Original Article

\title{
Effects of the Electrode Type on N100 and P300 in tDCS Applications
}

\author{
Jeong-Woo Lee, PhD ${ }^{1)}$, Se-Won Yoon, $\mathrm{PhD}^{1)}$, Woong-Sik Park, $\mathrm{PhD}^{2}$, Myoung Heo, $\mathrm{PhD}^{3)}$, \\ Dong-Geol Lee, $\mathrm{PhD}^{4)}$, SeOng-Kwan JeOng, $\mathrm{PhD}^{5)^{*}}$ \\ 1) Department of Physical Therapy, Kwangju Women's University, Republic of Korea \\ 2) Department of Occupational Therapy, Kwangju Women's University, Republic of Korea \\ 3) Department of Occupational Therapy, Gwangju University, Republic of Korea \\ 4) Department of Physical Therapy, Chungnam University Hospital, Republic of Korea \\ 5) Department of Physical Therapy, Seoul Orthopedic Medicine: Munhwa-dong, Jung-gu, Republic of \\ Korea
}

\begin{abstract}
Purpose] This study investigated the effects of types of electrode on N100 and P300 in transcranial direct current stimulation (tDCS) applications. [Subjects and Methods] Thirty subjects were randomly assigned to two groups with 15 subjects in each group depending on the electrode types. A positive electrode on the primary motor area (C4) and a negative electrode on the left primary motor area (C3), and stimulation was applied for 20 minutes. Before and after tDCS, N100 and P300 were measured by attaching an electrode to Fp1 and Fp2. [Results] In tDCS applications, N100 and P300 showed no significant interaction effects between time and group for either latency or amplitude in the Fp1 and Fp 2 areas, but there was a statistically significant difference in the main effect duration. [Conclusion] The latencies of N100 and P300 were shortened and that their amplitudes increased in both the Fp1 and Fp2 areas, regardless of the type of electrode.

Key words: Transcranial direct current stimulation (tDCS), N100, P300
\end{abstract}

(This article was submitted Jan. 28, 2014, and was accepted Mar. 17, 2014)

\section{INTRODUCTION}

Transcranial direct current stimulation (tDCS) can be utilized as a key tool because it can affect the neural plasticity of the cerebral cortex. Furthermore, it can directly change the excitability of electrical potentials in a stimulated region, as well as indirectly affect the excitability of the same region at the other side of the cerebrum ${ }^{1}$. Such tDCS can also cause polarity-dependent changes in the excitability of the cerebral motor cortex ${ }^{2}$. Positive-polarity stimulation increases excitability as depolarization occurs. Negative-polarity stimulation, on the other hand, decreases excitability due to hyperpolarization occurring at the neurons or neural networks in corresponding regions. Moreover, a low intensity current affects excitability at the motor cortex $^{3)}$

The study conducted by Fregni et al. ${ }^{4)}$ revealed that tDCS improved working memory performance in a consecutive sequential letter-matching test, while Antal et al. ${ }^{5)}$ showed in their study that tDCS influenced visual cognitive function in the contrast sensitivity test, a visual function

*Corresponding author. Seong-Kwan Jeong (E-mail: skjsk70@hanmail.net)

(C2014 The Society of Physical Therapy Science. Published by IPEC Inc. This is an open-access article distributed under the terms of the Creative Commons Attribution Non-Commercial No Derivatives (by-ncnd) License $<$ http://creativecommons.org/licenses/by-nc-nd/3.0/> . test tDCS-related studies have mostly used carbon rubber electrodes in applications involving scalp electrodes. Carbon rubber electrodes are very inconvenient to use and are disliked by subjects because the face must be wrapped using separate straps, to keep the electrodes in place. Furthermore, the available electrodes are too large to stimulate specific cerebral areas. Therefore, this study was conducted to investigate the effects of electrode type on N100 and P300 when tDCS was applied to a primary motor area.

\section{SUBJECTS AND METHODS}

This study selected 30 healthy adult females in their 20's who had a non-dominant left hand. We obtained approval for this experiment from the research ethics committee of Kwangju Women's University. The subjects were randomly divided into two groups comprised 15 women each. Group I were administered tDCS with carbon rubber electrodes, while round, self-adhesive electrodes were used for Group II. The general features of the subjects are presented in Table 1.

tDCS was applied using an Endomed 482 (Enraf-Nonius B.V., Rotterdam, Netherlands). In accordance with the international 10-20 system placement method, the positive electrode was placed over the primary motor cortex at $\mathrm{C} 4$, and the negative electrode was placed at $\mathrm{C} 3$. The pulse duration was set to $2 \mathrm{~ms}$, and the interpulse duration to $5 \mathrm{~ms}$. The entire duration was set to last for a total of 20 minutes.

Carbon rubber electrodes $4 \times 6 \mathrm{~cm}^{2}$ (Daeyang Medical, 
Table 1. General characteristics of the subjects

\begin{tabular}{lcc}
\hline & Group I $(\mathrm{n}=10)$ & Group II $(\mathrm{n}=10)$ \\
\hline Age (years) & $20.6 \pm 0.5$ & $21.5 \pm 1.1$ \\
Height $(\mathrm{cm})$ & $161.3 \pm 3.7$ & $162.1 \pm 1.5$ \\
Weight $(\mathrm{kg})$ & $55.1 \pm 8.6$ & $56.6 \pm 7.1$ \\
\hline Mean \pm SD. & & \\
Group I: Carbon rubber electrode & \\
Group II: A circular adhesive electrode &
\end{tabular}

Wonju, South Korea) were used for experimental group I with a current intensity of $0.058 \mathrm{~mA} / \mathrm{cm}^{2}$, and $1.77 \mathrm{~cm}^{2}$ round, self-adhesive electrodes (SKINTACT, Leonhard Lang GmbH, Innsbruck, Australia) were used for experimental group II with a current intensity of $0.06 \mathrm{~mA} / \mathrm{cm}^{2}$.

To measure event-related brain potentials (ERP), an EEG-8 electroencephalograph (LXE5208, LAXTHA, Daejeon, South Korea) was used, and the TeleScan software (LAXTHA, Daejeon, South Korea) program was used to conduct the latency and amplitude analyses of N100 and P300. The sampling rate was set to $256 \mathrm{~Hz}$, and band-pass filtered between $1 \sim 50 \mathrm{~Hz}$. The attachment regions were wiped clean with alcohol to reduce regional resistance to less than $5 \mathrm{k} \Omega$ before the electrodes were attached. The Ag$\mathrm{AgCl}$ electrodes were attached to the $\mathrm{Fp} 1$ and $\mathrm{Fp} 2$ areas; the active electrode was placed on the right mastoid, and the earth electrode was placed on the left mastoid, in accordance with the international 10-20 system placement method.

The ERP, which is the average of brainwaves, appears after stimulating the targets, was analyzed based on the maximum negative potential value observed between 80 and $120 \mathrm{~ms}$ for N100 and on the maximum positive potential value between 250 and $500 \mathrm{~ms}$ for P300. A serial reaction time task was implemented. Arrow shapes were randomly displayed in two different colors on a monitor. The subjects were requested to move a blue-colored arrows in the arrow direction by their non-dominant left hand, and the red-colored arrows in the direction away from the arrow. Visual stimulation was presented a total of 50 times, among which 18 times were designated for target stimulation (red arrow) and 32 times for non-target stimulation (blue arrow). The temporal interval between each stimulation was set at 1 second, and each time duration was randomly presented between 2 to 4 seconds. Statistical analysis was performed with the SPSS 12.0 for windows. Repeated measures ANOVA was used to analyze the changes in the two groups according to the duration of treatment. A significance level of $\alpha=0.05$ was chosen.

\section{RESULTS}

The results of the experiment show that the N100 latency and amplitude had statistically insignificant differences in the interactions between time and group in both the Fp1 and $\mathrm{Fp} 2$ areas. However, a statistically significant difference was found in the main effect duration $(p<0.05)$ in both areas (Table 2). Thus, tDCS application reduced the N100 latency and increased its amplitude, regardless of the type of electrode. The P300 latency and amplitude had statistically insignificant differences in interactions between time and group in both the Fp1 and Fp2 areas. However, a statistically significant difference was found in main effect duration $(\mathrm{p}<0.05)$ in both areas (Table 3$)$. Thus, tDCS application reduced the P300 latency and increased its amplitude, regardless of the electrode type.

\section{DISCUSSION}

Utz et al. ${ }^{6}$ reported that generalized electrode sizes had yet to be established for tDCS; however, some researchers have recommended using small electrodes to effectively transmit current. Our present results demonstrate that tDCS shortens the latencies and increases the amplitudes of N100 and P300 at both the Fp1 and Fp2 areas, regardless of the electrode type. N100 reflects early attentiveness in a cerebral process ${ }^{7}$; therefore, it considered to be an indicator of selective attentiveness ${ }^{8}$. In addition, N100 latency represents the complexity and efficiency of synapses involved in information processing speed and responses ${ }^{9}$. Therefore, we consider tDCS affected N100 latency and amplitude and to have aided the neurophysiologic processes that are related to attentiveness, information processing speed, and the responses of the subjects to external stimuli.

Hwang and Lee ${ }^{10)}$ reported that tDCS delivered by carbon rubber electrodes to stroke patients' primary motor cortex resulted in shorter P300 latency at both the Fp1 and 2 areas. Their result is similar to the result our present study, which used healthy people as subjects. The P300 amplitude represents the amount of cognitive activity, such as memory and attentiveness, and is related to the time it takes to evaluate and classify latencies in response to stimulation ${ }^{11}$. In addition, P300 also represents the information-gathering process of perceptual decisions that identify and determine stimulation from outside influences ${ }^{12,13)}$. Therefore, given that both N100 and P300 latencies were shortened, and that their amplitudes increased, regardless of the electrode type, we consider tDCS increased cognitive activities that execute tasks in response to target and non-target stimulation, regardless of the electrode type. Consequently, since tDCS positively influenced cognitive activities, regardless of the electrode type, we consider self-adhesive electrodes are a better treatment choice because of their user-friendliness and convenience in clinical applications of tDCS compared with carbon rubber electrodes. 
Table 2. Changes in N100

\begin{tabular}{cccccc}
\hline \multirow{2}{*}{ EEG } & Group & \multicolumn{2}{c}{ Latency $(\mathrm{sec})$} & \multicolumn{2}{c}{ Amplitude $(\mu \mathrm{V})$} \\
\cline { 3 - 6 } & & Pre & Post & Pre & Post \\
\hline \multirow{2}{*}{ Fp1 } & I & $0.14 \pm 0.04$ & $0.13 \pm 0.04$ & $-6.37 \pm 6.91$ & $-7.28 \pm 7.58$ \\
& II & $0.13 \pm 0.03$ & $0.11 \pm 0.04$ & $-7.58 \pm 9.40$ & $-8.50 \pm 9.88$ \\
Fp2 & I & $0.14 \pm 0.04$ & $0.13 \pm 0.03$ & $-6.71 \pm 7.23$ & $-7.91 \pm 7.07$ \\
& II & $0.14 \pm 0.03$ & $0.12 \pm 0.03$ & $-7.49 \pm 9.13$ & $-8.95 \pm 8.16$ \\
\hline
\end{tabular}

Mean \pm SD. At Fp1 and Fp2, there were only significant changes depending on time in the latency $(\mathrm{p}<0.05)$ and amplitude $(\mathrm{p}<0.05)$, but there was no statistically significant difference in interaction effect.

Group I: Carbon rubber electrode

Group II: A circular adhesive electrode

Table 3. Changes in $\mathrm{P} 300$

\begin{tabular}{cccccc}
\hline \multirow{2}{*}{ EEG } & \multirow{2}{*}{ Group } & \multicolumn{2}{c}{ Latency $(\mathrm{sec})$} & \multicolumn{2}{c}{ Amplitude $(\mu \mathrm{V})$} \\
\cline { 3 - 6 } & & Pre & Post & Pre & Post \\
\hline \multirow{2}{*}{ Fp1 } & I & $0.34 \pm 0.06$ & $0.32 \pm 0.05$ & $12.35 \pm 8.70$ & $13.51 \pm 10.45$ \\
& II & $0.33 \pm 0.08$ & $0.32 \pm 0.09$ & $13.87 \pm 15.04$ & $14.27 \pm 14.97$ \\
\multirow{2}{*}{ Fp2 } & I & $0.34 \pm 0.04$ & $0.31 \pm 0.04$ & $13.51 \pm 10.45$ & $17.52 \pm 14.05$ \\
& II & $0.33 \pm 0.09$ & $0.31 \pm 0.10$ & $15.27 \pm 15.11$ & $18.05 \pm 18.29$ \\
\hline
\end{tabular}

Mean $\pm \mathrm{SD}$. At $\mathrm{Fp} 1$ and $\mathrm{Fp} 2$, there were only significant changes depending on time in the latency $(\mathrm{p}<0.05)$ and amplitude $(\mathrm{p}<0.05)$, but there was no statistically significant difference in interaction effect.

Group I: Carbon rubber electrode

Group II: A circular adhesive electrode

\section{REFERENCES}

1) Vines BW, Nair DG, Schlaug G: Contralateral and ipsilateral motor ef fects after transcranial direct current stimulation. Neuroreport, 2006, 17: 671-674. [Medline] [CrossRef]

2) Nitsche MA, Doemkes S, Karaköse T, et al.: Shaping the effects of transcranial direct current stimulation of the human motor cortex. J Neurophysiol, 2007, 97: 3109-3117. [Medline] [CrossRef]

3) Furubayashi T, Terao Y, Arai N, et al.: Short and long duration transcranial direct current stimulation (tDCS) over the human hand motor area. Exp Brain Res, 2008, 185: 279-286. [Medline] [CrossRef]

4) Fregni F, Boggio PS, Nitsche M, et al.: Anodal transcranial direct current stimulation of prefrontal cortex enhances working memory. Exp Brain Res, 2005, 166: 23-30. [Medline] [CrossRef]

5) Antal A, Nitsche MA, Paulus W: External modulation of visual perception in humans. Neuroreport, 2001, 12: 3553-3555. [Medline] [CrossRef]

6) Utz KS, Dimova V, Oppenländer K, et al.: Electrified minds: transcranial direct current stimulation (tDCS) and galvanic vestibular stimulation (GVS) as methods of non-invasive brain stimulation in neuropsychologya review of current data and future implications. Neuropsychologia, 2010,
48: 2789-2810. [Medline] [CrossRef]

7) Kim MS, Kwon JS, Kim JJ: The neurophysiological mechanism of working memory: an event-related potential study. Kor J Psychol, 2004, 23 : 313-326.

8) Hillyard SA, Hink RF, Schwent VL, et al.: Electrical signs of selective attention in the human brain. Science, 1973, 182: 177-180. [Medline] [CrossRef]

9) Boutros NN, Korzyuko O, Oliwa G, et al.: Morphological and latency abnormalities of the mid-latency auditory evoked responses in schizophrenia: a preliminary report. Schizophr Res, 2004, 70: 303-313. [Medline] [CrossRef]

10) Hwang KK, Lee JW: Effect of applying tDCS by inactive electrode placement to cognitive response on stroke patients. J Korean Acad Clin Elec, 2013, 11: 31-38. [CrossRef]

11) Polich J: Clinical application of the P300 event-related brain potential Phys Med Rehabil Clin N Am, 2004, 15: 133-161. [Medline] [CrossRef]

12) Kwon JS: The use of event-related potentials in the study of cognitive functions. J Cogn Sci, 2000, 1: 79-98.

13) Sutton S, Braren M, Zubin J, et al.: Evoked-potential correlates of stimulus uncertainty. Science, 1965, 150: 1187-1188. [Medline] [CrossRef] 